\title{
Caracterización y propuesta de formulación de los contenidos curriculares de la química en el ciclo cuatro
}

Ballesteros Hurtado Jenny' y Suarez Cerpa Beris²

Categoría 2. Trabajo de investigación (en proceso)

\section{Resumen}

La presente comunicación pretende mostrar los avances logrados en un trabajo de investigación que se adelanta en la Maestría en Docencia de la Química de la UPN de Colombia, enfocado en la caracterización de los contenidos curriculares del ciclo cuatro (grados octavo y noveno) de las Instituciones educativas Colegio Canapro e Instituto Educativo Distrital Brasilia de la Ciudad de Bogotá. La investigación incluye una indagación conceptual y metodológica frente a los estudios realizados sobre contextualización de los contenidos curriculares en química en el ámbito local e internacional, que permitieron el planteamiento de las categorías de análisis de esta investigación y la utilización de instrumentos de recolección de información, tales como encuestas y entrevistas a docentes del área de ciencias y estudiantes, observación de clases y de reuniones de área, que permita conocer el estado actual de las instituciones frente a lo que se tiene y a la importancia en el aprendizaje de los contenidos curriculares.

Es una investigación cualitativa, con un enfoque de estudio de caso y con la modalidad de estudio instrumental y descriptivo. Se desarrollará en tres fases que incluyen la caracterización de los contenidos curriculares, el planteamiento de la propuesta y la evaluación de la misma.

\section{Palabras claves}

Currículo, contenidos curriculares, contextualización.

\footnotetext{
'Estudiante de la Maestría en Docencia de la Química. Universidad Pedagógica Nacional. jennyballesteros@gmail.com

2Estudiante de la Maestría en Docencia de la Química Universidad Pedagógica Nacional. erpa2602@gmail.com
} 
Revista Tecné, Episteme y Didaxis: TED. Año 2014, Número Extraordinario. ISSN Impreso: 0121-3814, ISSN web: 2323-0126

Memorias, Sexto Congreso Internacional sobre Formación de Profesores de Ciencias. 08 al 10 de octubre de 2014, Bogotá

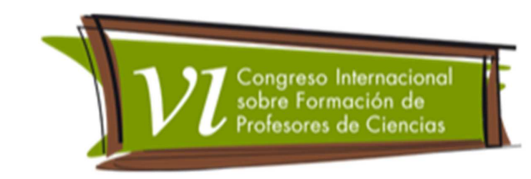

\section{Objetivo general}

El trabajo que se presenta pretende caracterizar los contenidos curriculares de la química del ciclo cuatro y a partir de esto desarrollar una propuesta que involucre las particularidades de las instituciones objeto de esta investigación (Colegio Canapro y I.E.D. Brasilia), es decir, proponer un diseño curricular contextualizado.

Por lo tanto se deben:

- Caracterizar los contenidos de un currículo contextualizado para la enseñanza de la química en estudiantes de ciclo cuatro

- Analizar la incidencia que han tenido los contenidos que hasta ahora se han trabajado en química en los estudiantes de ciclo 4 y en los docentes del área de ciencias naturales.

- Identificar las características epistemológicas, sociales, didácticas y los requerimientos de un currículo contextualizado para la enseñanza de la química en estudiantes de ciclo cuatro en las instituciones objeto de estudio.

\section{Contextualización curricular}

Se puede afirmar que la contextualización curricular permite articular el conocimiento científico escolar con los conocimientos de los estudiantes haciendo que ellos apliquen lo que aprenden en la escuela hacia otros contextos sociales (Fernandeset al., 2011).

En la actualidad, la educación química se centra en seleccionar contenidos que deben estar presentes en un currículum equilibrado de química, mejorar la comprensión de los conceptos y modelos químicos, contextualizar los contenidos, de manera que los estudiantes puedan adquirir conciencia de la utilidad y aplicabilidad de lo que ellos aprenden, y promover una mejor apreciación de la naturaleza de la química y sus implicaciones sociales. Además de esto, se investigan nuevas estrategias didácticas que tengan en cuenta las dificultades de aprendizaje de los estudiantes, su motivación y el forjamiento de competencias ciudadanas, entre otros.

Por otra parte, la actualización de los contenidos del currículum de química en la educación secundaria está siendo abordada con diferentes estrategias y ámbitos 
Revista Tecné, Episteme y Didaxis: TED. Año 2014, Número Extraordinario. ISSN Impreso: 0121-3814, ISSN web: 2323-0126

Memorias, Sexto Congreso Internacional sobre Formación de Profesores de Ciencias. 08 al 10 de octubre de 2014, Bogotá

en cada país, si bien puede afirmarse que es un objetivo claramente establecido en la comunidad de educadores, formadores y planificadores del currículo de química, e investigadores en didáctica de la química. Contextualizar el currículum de química significa usar los contextos y las aplicaciones de la química como medio de desarrollar los conceptos e ideas de la ciencia o de justificar su importancia, además y de acuerdo con Mallarino (2007) debe tener en cuenta la multiculturalidad, ser interdisciplinar, siempre involucrando la realidad en lo que se quiere incluir.

Para conseguir un currículum de química más relevante y acorde con las finalidades de la educación científica, autores como Gabel (1998), Caamaño (2001a), Caamaño e Izquierdo (2007), Gómez Crespo et al., (2003) y Mahaffy, (2004) manifiestan que debe replantearse gran parte de los contenidos actuales de la química, evaluando su relevancia en función de las finalidades de la educación en ciencias.

De otro lado, y siguiendo lo planteado de Mora y Parga (2007) sobre las tramas histórico- epistemológicas se debe articular el conocimiento del profesorado al diseño curricular, como otro mecanismo de contextualización, lo que genera la necesidad de construir tramas y unidades didácticas, además de la implicación con el conocimiento didáctico del contenido, que se hace indispensable a la hora de formar profesores.

\section{La educación química para qué}

Una forma de lograr que los estudiantes se interesen por el estudio de la ciencia, es guiarlos a que conozcan el valor socio-cultural de la misma, generando actitudes en beneficio de sí mismos y de la sociedad a la cual pertenecen, y es el docente quien tiene bastante influencia para alcanzar este objetivo, teniendo presente la relevancia de la motivación como estrategia para alcanzar un verdadero aprendizaje. Por tanto el currículo debe incluir creencias, actitudes y valores que forme sujetos participes de la transformación y el mejoramiento de su entorno (Mora y Parga, 2010).

\section{Problema de Investigación}

¿Qué cambios y estrategias se deben implementar en el plan curricular de las instituciones objeto de estudio para lograr una formación integral, que 
Revista Tecné, Episteme y Didaxis: TED. Año 2014, Número Extraordinario. ISSN Impreso: 0121-3814, ISSN web: 2323-0126

Memorias, Sexto Congreso Internacional sobre Formación de Profesores de Ciencias. 08 al 10 de octubre de 2014, Bogotá

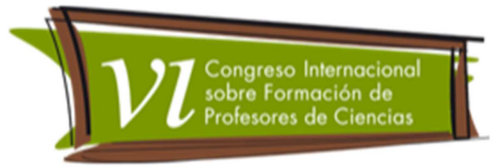

corresponda a los requerimientos sociales y personales del estudiante, desarrollando contenidos en química para ciclo 4 contextualizados?

\section{Metodología de investigación}

La metodología de la investigación a desarrollar es cualitativa, y está fundamentada en una perspectiva interpretativa que permite comprender las acciones, mejoras y transformaciones de los contenidos en contexto abordados con los estudiantes, por lo que la hace naturalista, sin alterar las condiciones del entorno, permitiendo que se comporten como lo hacen en su vida cotidiana (Hernández, et al., 2006).

Se realizará mediante el enfoque de estudio de casos ya que es un método que tiene en cuenta la particularidad y la complejidad de la problemática escolar a tratar, permitiendo la comprensión de la misma.

Se llevará a cabo en tres fases: una fase inicial general, que incluye la caracterización de las instituciones educativas participantes, partiendo de la revisión de los resultados de las pruebas de evaluación externa aplicadas a los estudiantes, y que plantean fortalezas y debilidades en las competencias que a nivel nacional deben tener para este ciclo. Se hará la identificación de la población, su estrato socio-económico, intereses, visión frente a la química y sus expectativas frente a la asignatura; en cuanto a los docentes se indagará sobre los criterios de selección de contenidos, el desarrollo de la clase a partir de lo programado en el plan de estudios y el punto de vista que ellos tienen de los procesos de enseñanza y aprendizaje de la ciencia. A partir de las categorías de análisis sobre las implicaciones que se deben tener en cuenta en la estructuración y diseño de los contenidos curriculares (Figura 1) se propuso la siguiente tabla:

Tabla 1. Categorías de análisis

\begin{tabular}{|l|l|l|l|}
\hline \multicolumn{1}{|c|}{ CATEGORIA } & SUBCATEGORIA & \multicolumn{1}{|c|}{ INDICADORES } & \multicolumn{1}{|c|}{$\begin{array}{l}\text { INSTRUMENTOS } \\
\text { EVIDENCIA }\end{array}$} \\
\hline $\begin{array}{l}\text { HISTORICA y } \\
\text { EPISTEMOLOGI } \\
\text { CA }\end{array}$ & $\begin{array}{l}\text { Evolución del } \\
\text { conocimiento }\end{array}$ & $\begin{array}{l}\text { Identificar el marco referencial y } \\
\text { teórico del docente en el proceso } \\
\text { de enseñanza. }\end{array}$ & $\begin{array}{l}\text { Análisis documental } \\
\text { (características del } \\
\text { texto empleado por el } \\
\text { docente, } \\
\text { implementación de los } \\
\text { estándares en el } \\
\text { currículo) en el currículo las }\end{array}$ \\
\hline
\end{tabular}


Revista Tecné, Episteme y Didaxis: TED. Año 2014, Número Extraordinario. ISSN Impreso: 0121-3814, ISSN web: 2323-0126

Memorias, Sexto Congreso Internacional sobre Formación de Profesores de Ciencias. 08 al 10 de octubre de 2014, Bogotá

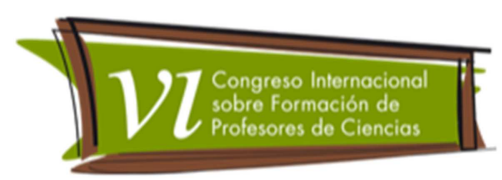

\begin{tabular}{|c|c|c|c|}
\hline & $\begin{array}{l}\text { Mecanismo de } \\
\text { producción del } \\
\text { conocimiento } \\
\text { químico }\end{array}$ & $\begin{array}{l}\text { Identificar las concepciones de } \\
\text { ciencia del docente. } \\
\text { Verificar los criterios de } \\
\text { construcción de la química y que } \\
\text { el docente tiene en cuenta para } \\
\text { el desarrollo de los contenidos } \\
\text { curriculares. }\end{array}$ & $\begin{array}{l}\text { Análisis documental } \\
\text { (Plan de estudios, } \\
\text { estándares } \\
\text { lineamientos } \\
\text { curriculares) } \\
\text { Encuesta } \\
\text { Entrevista } \\
\text { estructurada, } \\
\text { observación de clase }\end{array}$ \\
\hline & $\begin{array}{l}\text { Obstáculos } \\
\text { epistemológicos }\end{array}$ & $\begin{array}{l}\text { Establecer el } \\
\text { conocimiento que el docente } \\
\text { tiene sobre los obstáculos } \\
\text { epistemológicos. } \\
\text { Identificar la relevancia que da el } \\
\text { docente a los obstáculos } \\
\text { epistemológicos } \\
\text { construcción del currículo. }\end{array}$ & $\begin{array}{l}\text { Entrevista semi- } \\
\text { estructurada. } \\
\text { Observación de clase. } \\
\text { Análisis documental. }\end{array}$ \\
\hline $\begin{array}{l}\text { CONOCIMIENT } \\
\text { O DISCIPLINAR } \\
\text { DEL } \\
\text { CONTENIDO } \\
\text { QUÍMICO }\end{array}$ & $\begin{array}{l}\text { Sustantivo y } / 0 \\
\text { declarativo }\end{array}$ & $\begin{array}{l}\text { Evidenciar el manejo disciplinar de } \\
\text { los contenidos curriculares por } \\
\text { parte del docente. } \\
\text { Identificar el uso de modelos y } \\
\text { analogías que hace el docente } \\
\text { para la enseñanza de los } \\
\text { contenidos. }\end{array}$ & $\begin{array}{l}\text { Análisis documental } \\
\text { (Diseños y } \\
\text { planeaciones), } \\
\text { Observación de clase }\end{array}$ \\
\hline $\begin{array}{l}\text { CONOCIMIENT } \\
\text { O DEL } \\
\text { CONTEXTO } \\
\text { ESCOLAR }\end{array}$ & $\begin{array}{l}\text { Conocimiento } \\
\text { del } \\
\text { direccionamiento } \\
\text { estratégico } \\
\text { institucional }\end{array}$ & $\begin{array}{l}\text { Identificar la inclusión de las } \\
\text { políticas y normas institucionales } \\
\text { en el plan de estudios y } \\
\text { planeador. }\end{array}$ & $\begin{array}{l}\text { Entrevista semi- } \\
\text { estructurada } \\
\text { Análisis documental, } \\
\text { Observación de clases }\end{array}$ \\
\hline & $\begin{array}{l}\text { Caracterización } \\
\text { de los estudiantes } \\
\text { y su entorno }\end{array}$ & $\begin{array}{l}\text { Verificar el conocimiento que el } \\
\text { docente tiene sobre el contexto } \\
\text { escolar. } \\
\text { Establecer el nivel de implicación } \\
\text { del contexto escolar en el diseño } \\
\text { curricular. }\end{array}$ & \\
\hline $\begin{array}{l}\text { PSICO - } \\
\text { PEDAGÓGICO } \\
\text { - DIDÁCTICO }\end{array}$ & $\begin{array}{l}\text { Aspectos } \\
\text { psicológicos, } \\
\text { pedagógicos y } \\
\text { didácticos en el } \\
\text { currículo. }\end{array}$ & $\begin{array}{l}\text { Analizar la implicación que se da } \\
\text { en el diseño curricular a las } \\
\text { concepciones alternativas del } \\
\text { estudiante. }\end{array}$ & $\begin{array}{l}\text { Entrevista semi- } \\
\text { estructura. } \\
\text { Análisis documental } \\
\text { Observación de clase }\end{array}$ \\
\hline
\end{tabular}


Revista Tecné, Episteme y Didaxis: TED. Año 2014, Número Extraordinario. ISSN Impreso: 0121-3814, ISSN web: 2323-0126

Memorias, Sexto Congreso Internacional sobre Formación de Profesores de Ciencias. 08 al 10 de octubre de 2014, Bogotá

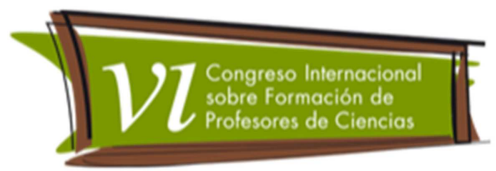

\begin{tabular}{|c|c|c|c|}
\hline & & $\begin{array}{l}\text { hace durante el diseño curricular } \\
\text { de la etapa del desarrollo de } \\
\text { pensamiento en el que se } \\
\text { encuentra el estudiante. }\end{array}$ & \\
\hline & $\begin{array}{ll}\text { Modelos de } & \text { de } \\
\text { enseñanza de los } \\
\text { docentes }\end{array}$ & $\begin{array}{l}\text { Reconocer las implicaciones de } \\
\text { los modelos didácticos en la } \\
\text { selección y enseñanza de los } \\
\text { contenidos curriculares. }\end{array}$ & $\begin{array}{l}\text { Análisis documental } \\
\text { (Plan de estudios y } \\
\text { planeador), } \\
\text { Observación de clase }\end{array}$ \\
\hline & & $\begin{array}{l}\text { Caracterizar las estrategias } \\
\text { didácticas empleadas por el } \\
\text { docente para facilitar el } \\
\text { aprendizaje de los contenidos } \\
\text { curriculares. }\end{array}$ & \\
\hline \multirow[t]{7}{*}{ SOCIAL } & Entorno cultural & $\begin{array}{l}\text { Indagar frente a la implicaciones } \\
\text { del entorno cultural en el currículo }\end{array}$ & $\begin{array}{l}\text { Entrevista } \\
\text { estructura. }\end{array}$ \\
\hline & & & Análisis documental \\
\hline & & & Observación de clase \\
\hline & Proyección social & $\begin{array}{lll}\text { Evaluar la incidencia de los } \\
\text { contenidos frente a los } \\
\text { requerimientos sociales y } \\
\text { proyectos de vida }\end{array}$ & $\begin{array}{l}\text { Análisis documental } \\
\text { Encuesta }\end{array}$ \\
\hline & Axiología & $\begin{array}{l}\text { Identificar la implicación de los } \\
\text { valores en la planeación } \\
\text { curricular. }\end{array}$ & $\begin{array}{l}\text { Análisis documental } \\
\text { Encuesta }\end{array}$ \\
\hline & $\begin{array}{l}\text { Aspectos } \\
\text { interdisciplinarios }\end{array}$ & $\begin{array}{l}\text { Reconocer las integraciones que } \\
\text { se establecen entre las diferentes } \\
\text { áreas con los contenidos de la } \\
\text { ciencia. }\end{array}$ & $\begin{array}{ll}\text { Entrevista } & \text { semi } \\
\text { estructurada } & \end{array}$ \\
\hline & CTS & $\begin{array}{l}\text { Evaluar si los contenidos } \\
\text { curriculares obedecen a } \\
\text { problemáticas reales del entorno } \\
\text { y/o de las relaciones CTS. } \\
\text { Identificar la inclusión de las } \\
\text { actitudes científicas y los avances } \\
\text { tecnológicos en la enseñanza. }\end{array}$ & $\begin{array}{l}\text { Análisis documental y } \\
\text { observaciones de } \\
\text { clase }\end{array}$ \\
\hline
\end{tabular}

Fuente. Mora y Parga, (2008) 
Revista Tecné, Episteme y Didaxis: TED. Año 2014, Número Extraordinario. ISSN Impreso: 0121-3814, ISSN web: 2323-0126

Memorias, Sexto Congreso Internacional sobre Formación de Profesores de Ciencias. 08 al 10 de octubre de 2014, Bogotá

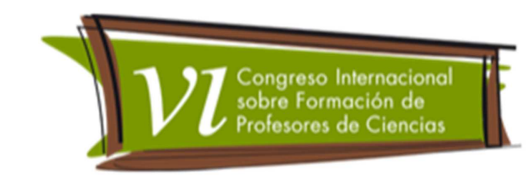

En la fase de diseño que se generará a partir del análisis e interpretación de la información de la primera fase, recogerá los factores que determinan el desarrollo del currículo, pertinencia de lo que actualmente tienen las instituciones en su plan de estudios y los aportes que partir de esta investigación se sugieren para el mejoramiento del proceso de aprendizaje. La última fase de evaluación de la propuesta del plan de estudios con los contenidos curriculares contextualizados, determinará su efectividad frente a las necesidades e intereses de los estudiantes, de la institución y de la sociedad, realizando un pilotaje para conocer la factibilidad y significado de la propuesta.

La recolección de la información se realizará a partir de entrevistas de tipo semiestructurado, encuestas, observación y análisis documental donde se involucra a los docentes del área de ciencias naturales y a algunos estudiantes.

La congruencia de los datos está determinada por la triangulación, ya que se permitirá la confrontación de las percepciones de los investigadores, lo que elevará el nivel de interpretación de los datos y de aportes para la reestructuración del currículo.

\section{Conclusiones}

- La construcción de instrumentos de recolección de la información, son determinantes para conocer el manejo e importancia de los contenidos curriculares que se da en las instituciones objeto de estudio, a partir de la visión de los estudiantes y los docentes.

- Las categorías de análisis permite conocer el estado actual de las instituciones educativas frente a los contenidos curriculares y además sirven de base para los aportes que esta investigación pretende dar al diseño curricular en el área de ciencias naturales, con relación a los contenidos curriculares contextualizados

- Los antecedentes permiten comprender los cambios de los contenidos curriculares que se hacen necesarios por los requerimientos sociales, donde se busca lograr que el estudiante le dé sentido a lo que aprende y recuperar la capacidad explicativa de la química, lo cual conlleva a replantear lo que hasta el momento se ha trabajado y darle un nuevo significado. 
Revista Tecné, Episteme y Didaxis: TED. Año 2014, Número Extraordinario. ISSN Impreso: 0121-3814, ISSN web: 2323-0126

Memorias, Sexto Congreso Internacional sobre Formación de Profesores de Ciencias. 08 al 10 de octubre de 2014, Bogotá

- La contextualización de los contenidos curriculares debe entenderse no solo en su relación con el entorno, sino además con aspectos históricos, epistemológicos, del conocimiento disciplinar del contenido químico, psicopedagógico, didáctico y social, que permiten plantear una propuesta en la formulación de los contenidos curriculares durante el diseño curricular.

\section{Referencias bibliográfica}

Caamaño, A. (2006). Retos del currículo de química en la educación secundaria. la selección y contextualización de los contenidos de química en los curriuclos de Inglaterra, Portugal, Francia y España. Educación Química N. 17.

Caamaño, A. (2007). Modelizar y contextualizar el currículo de química: un proceso en constante desarrollo. En M. Izquierdo, A. Caamaño, \& M. Quintanilla, Investigar en la enseñanza de la química. Nuevos horizontes: contextualizar y modelizar (pág. 19-34). Barcelona: Universidad autonoma de Barcelona.

Fernandes, P., Leite, C., Mouraz, A., Figueiredo, C. (2011). Significados atribuidos al concepto de contextualización curricular. XI congreso internacional Gallego - Portugues de psicopedagogía. Coruña.

Hernández, R. (2010).Metodología de la investigación. Bogotá, Mac Graw Hill.

Mallarino, C. (2007). La contextualización del currículo: cognición y no verbalidad. Revista científica Guillermo de Ockham. pág. 73-84.

Mora, W. M., Parga, D. (2010). La imagen pública de la química y su relación con la generación de actitudes hacia la química y su aprendizaje. Revista Tecné, Episteme y Didaxis: TED. 27 pág. 67-93.

Mora, W., Parga, D. (2007). Tramas histórico-epistemológicas en la evolución de la teoría estructural en química orgánica. Revista Tecné, Episteme y Didaxis: TED. 21, p. 100-118. 